\title{
Influências Subjacentes à Escolha da Carreira por Psicólogos Clínicos e Não Clínicos
}

\section{Influences Underlying Career Choice by Clinical and Non-Clinical Psychologists}

\author{
Liliana Sofia Reis Rebelo, Maria da Luz Vale-Dias \\ Universidade de Coimbra
}

\begin{abstract}
Resumo
Este estudo teve como objetivo perceber quais as influências que originam a escolha da carreira em Psicologia e verificar se o trauma ou situações adversas contribuem para essa mesma escolha. A amostra incluiu 204 sujeitos com formação em Psicologia que foram divididos em dois grupos, os da área Clínica e da área Não Clínica. Foram utilizados como instrumentos o Questionário Influences on Becoming a Therapist-IBT, adaptado para Portugal na presente pesquisa, a EADS-21, a ESAF e a ECA. Os resultados indicam que antecedentes de problemas pessoais não são universais entre pessoas que se tornam Psicólogos.

Palavras chave: Influências, Escolha da Carreira, Centralidade do Evento Traumático, Sintomas Emocionais, Felicidade.
\end{abstract}

\begin{abstract}
This study intended to investigate the influences that accompany someone's career choice in Psychology, as well as to verify if trauma or adverse experiences contribute to this same choice. The sample included 204 subjects with professional training in Psychology. Subjects were divided into two groups: Clinical Psychologists and Non-Clinical Psychologists. Data collection was carried out by applying the Portuguese adaptation of Influences on Becoming a Therapist-IBT, performed in this research, the EADS-21 scale, the Happiness scale, and also the Centrality of Event Scale. The results indicate that antecedents of personal problems are not universal among people who become Psychologists.

Keywords: Influences, Career Choice, Centrality of Traumatic Event, Emotional Symptoms, Happiness.
\end{abstract}

Como descrito por Flannery (1999), o trauma psicológico diz respeito ao "impacto de um (ou mais) acontecimento(s) crítico(s) no funcionamento psicológico ou biológico de um indivíduo". Considerando que as memórias de acontecimentos emocionais são uma parte importante da nossa história de vida e identidade, algumas destas memórias podem continuar a provocar sofrimento ao longo da existência (Bluck \& Habermas, 2000; McAdams, 2001; Pillemer, 1998; Singer \& Salovey, 1993 citados em Matos, Pinto-Gouveia \& Gomes, 2010). Muitos investigadores, nesta área, afirmam que as experiências traumáticas geram um desequilíbrio acentuado na mente das vítimas, deixando os esquemas mentais destas danificados. Este processo torna as experiências traumáticas difíceis de processar e, por sua vez, estas tornam-se pobremente integradas nas auto-narrativas do sujeito (Horowitz, 1986; Van der Kolk \& Fisler, 1995, citados por Bluck, 2003). No entanto, Berntsen e Rubin (2006, 2007) desenvolveram a teoria da Centralidade do Evento, na qual defendem exatamente o oposto: que os desvios nos esquemas mentais não levam a memórias pobres ou fragmentadas. Pelo contrário, em vez de se encontrarem pobremente integradas, as memórias traumáticas (devido ao seu impacto emocional), em muitos casos, ficam altamente acessíveis e podem formar um ponto de referência cognitivo para a organização do conhecimento autobiográfico, com um impacto contínuo na interpretação de experiências não traumáticas e expetativas para o futuro, e este parece ser o ponto de partida para explicar a forma como eventos traumáticos afetam a memória e o conhecimento.

Mesmo assim, pessoas que sofrem eventos negativos parecem capazes de processar satisfatoriamente esses eventos, a nível emocional e cognitivo. Por vezes, essas ocorrências relacionam-se com escolhas bem adaptadas.

A literatura tem sugerido que, por vezes, terapeutas seguem as suas profissões na tentativa de resolver problemas psicológicos pessoais (Guy, 1987; Henry, Sims \& Spray, 1971; Sussman, 1992 citados em Murphy \& Halgin, 1995), para satisfazer necessidades próprias não conhecidas ou não resolvidas (Guy \& Liaboe, 1986; Guy, Poelstra \& Strak, 1989 citados em Murphy \& Halgin, 1995), para resolver questões relativas aos problemas nas suas famílias de origem (Elliot \& Guy, 1993; Fussell \& Bonney, 1990; Guy, Tamura \& Poelstra, 1989; Henry, Sims \& Spray, 1971; Liaboen \& Guy, 1987; Racusin, Abramowitz \& Winter, 1981; Sussman, 1992 citados em Murphy \& Halgin, 1995), ou pela necessidade de dar continuação ao papel de "cuidador" que tinham na família de origem (DiCaccavo, 2002). Neste âmbito, os objetivos desta investigação passam por: (1) aceder a fatores que estão na base da escolha da carreira em Psicologia, e (2) verificar se o trauma ou situações adversas contribuem para a escolha da carreira em Psicologia e se afetam mais os profissionais de Psicologia Clínica. 


\section{Método}

\section{Participantes}

A amostra, de conveniência, é composta por 204 sujeitos, sendo $92.2 \%$ do sexo feminino e $7.8 \%$ do masculino, com idades compreendidas entre os 22 e os 60 anos $(M=33 ; \mathrm{DP}=10.11)$. Os sujeitos têm formação profissional em Psicologia e foram divididos em dois grupos, os da área Clínica $(72.5 \%, \mathrm{~N}=148)$ e os da Não Clínica $(27.5 \%, \mathrm{~N}=56)$.

\section{Instrumentos}

Para a recolha de dados foi inicialmente administrado um consentimento informado, um questionário sociodemográfico e, ainda, mais quatro instrumentos. $\mathrm{O}$ Questionário sobre as Influências na Escolha da Carreira de Psicologia, adaptação portuguesa (Rebelo \& Vale Dias, 2016) de Influences on Becoming a Therapist-IBT (Murphy \& Halgin, 1995), avalia as influências na escolha de carreira e o impacto dessas influências no funcionamento profissional. A versão original apresentou um Alfa de Cronbach de .85 e é constituída por 36 itens, na sua maioria de resposta fechada, numa escala tipo Likert de 4 pontos ( $3=$ Concordo totalmente a $0=$ Discordo/Neutralidade). A versão portuguesa realizada neste estudo, de 29 itens, apresenta um alfa total de .88 .

A EADS-21 (Pais-Ribeiro, Honrado e Leal, 2004), versão portuguesa da Depression Anxiety Stress Scales (DASS; Lovibond e Lovibond, 1995), utilizada neste estudo como medida de Sintomas Emocionais Negativos, abreviadamente Sintomas, organiza-se em três escalas: Depressão $(\alpha=.85)$, Ansiedade $(\alpha=.74)$ e Stress $(\alpha=.81)$, cada uma delas composta por sete itens. Cada item consiste numa afirmação, que remete para sintomas emocionais negativos. Neste estudo, as três escalas apresentam bons índices de fiabilidade: Depressão $(\alpha=.85)$, Stress $(\alpha=.85)$ e Ansiedade $(\alpha=.74)$.

A Escala sobre a Felicidade - ESAF (Barros, 2001) reveste-se de um caráter eclético abrangente às diversas dimensões da felicidade, bem-estar e satisfação com a vida. Neste estudo, a escala apresenta um excelente Alfa de Cronbach $(\alpha=.94)$, superior ao original $(\alpha=.85)$.

A Escala de Centralidade do Evento - ECA (Matos, Pinto-Gouveia, \& Gomes, 2010) é a versão portuguesa da Centrality of Event Scale (CES; Berntsen, \& Rubin, 2006). Este instrumento pretende avaliar a centralidade de um acontecimento (traumático) na identidade e na história de vida de uma pessoa. Este questionário de auto-relato é composto por 20 itens, que remetem para a noção de um acontecimento traumático (ou a sua memória) se constituir como um ponto de referência para a formação de expectativas e atribuição de significado a outros acontecimentos, como um ponto de viragem na história de vida e como central na identidade pessoal. Neste estudo, a escala apresenta um índice de fiabilidade excelente $(\alpha=.95)$, próximo do de $2010(\alpha=.96)$.

\section{Procedimentos}

Os dados foram recolhidos, de setembro a dezembro de 2016, através de questionários online, garantindo-se anonimato. Recorreu-se ao programa informático SPSS
(Statistical Package for the Social Sciences) versão 22.0 para a análise dos dados. Foi testada a normalidade das distribuições para cada escala, através do Teste de Kolmogorov-Smirnov. Neste sentido, foi possível concluir que as variáveis seguem uma distribuição normal $(p>.05)$, exceto na EADS-21 $(p<.05)$, tendo-se optado por testes paramétricos, ao considerar também o Teorema do Limite Central (quando $\mathrm{N} \geq 30$ ). O presente estudo examinou ainda as propriedades psicométricas da versão portuguesa da Escala IBT (Murphy \& Halgin, 1995) numa amostra da população com habilitação em Psicologia. O processo de tradução e adaptação da IBT seguiu os procedimentos habituais descritos na literatura (Behling, \& Law, 2000). A validade de constructo foi examinada com recurso à Análise em Componentes Principais (ACP).

A consistência interna e fiabilidade das diferentes escalas, incluídas na investigação, foram testadas através do Alfa de Cronbach. De forma a testar as associações entre as variáveis, foi utilizado o Coeficiente de Correlação de Pearson. Para testar o papel preditivo de algumas variáveis, recorreu-se ao Modelo de Regressão Linear Simples, estudando a relação entre uma variável independente contínua e as variáveis dependentes.

\section{Resultados}

$\mathrm{Na}$ análise prévia da dimensionalidade da IBT, efetuou-se uma ACP, cujos resultados finais não suportaram a estrutura original baseada em sete fatores, mas $\operatorname{sim}$ a existência de dois, Experiências $(\alpha=.86)$ e Motivações $(\alpha=.84)$, dimensões estas também teoricamente referidas pelo autor original. Esta solução apresenta bons indicadores de adequação da matriz $[$ Kaiser-Meyer-Olkin $-\mathrm{KMO}=.805$; e índice de esfericidade de Barttlet [ $\chi 2(630)=2659.952, p \leq .001]$.

Numa primeira análise de dados, foi testada a relação entre a Experiência de ausência ou perda dos pais durante a adolescência/juventude (por exemplo, doença, divórcio, separação, morte), Centralidade do Evento, Sintomas Emocionais Negativos e Felicidade nos grupos de psicólogos clínicos e não clínicos. $\mathrm{Na}$ amostra de Psicólogos Clínicos verificou-se que a correlação entre a Experiência de ausência ou perda dos pais e a ECA é baixa, mas significativa $(r=.21 p=.01)$. A subescala Depressão da EADS apresenta uma correlação negativa moderada $(r=-.35)$ e significativa com a $\operatorname{ESAF}(p \leq .001)$. As correlações da Ansiedade, subescala da EADS, com a ESAF e ECA são baixas, sendo a primeira negativa ( $r=-.19$ e $r=.25$, respetivamente), e significativas ( $p=.02 \mathrm{e}$ $p \leq .001$, respetivamente). Também o Stress (EADS) apresenta correlações baixas e estatisticamente significativas com a ESAF e ECA ( $r=-.23, p=.01$ e $r=.18$, $p=.03$, respetivamente). Na amostra de Psicólogos Não Clínicos existe uma correlação moderada entre a EADS Total e a ECA $(r=.36, p=.01)$, e também a subsescala da EADS intitulada Depressão se encontra moderadamente correlacionada com a ECA $(r=.41, p \leq .001)$.

Numa segunda análise, foram investigadas as associações da Centralidade do Evento Traumático e das Motivações (in Influências -IBT) com as Áreas de Especialização em Psicologia. Calculou-se uma 
ANOVA para analisar se existiam diferenças na Centralidade do Evento nos grupos de Psicólogos, Clínicos e Não Clínicos (cf. Tabela 1), verificando-se que não existem diferenças estatisticamente significativas ao nível da Centralidade do Evento ( $p$ >.05) segundo a área de especialização (cf. Tabela 1).

Tabela 1.

Comparação de médias (ANOVA) da Centralidade do Evento por área de especialização (Clínica e Não Clínica)

\begin{tabular}{ccccc}
\hline${ }^{1}$ ECA_Total & M & DP & $P$ & $F$ \\
\hline Clínica & 59.05 & 18.03 & \multirow{2}{*}{.35} & .88 \\
Não Clínica & 56.34 & 19.39 & &
\end{tabular}

Nota: ${ }^{1}$ Escala da Centralidade do Evento

Foram ainda comparadas as pontuações dos dois grupos de psicólogos nos itens que reportam ao Desejo de ajudar a sociedade e ao Desejo de ser professor (Motivações) (cf. Tabela 2). Não se registaram diferenças estatisticamente significativas entre os Psicólogos relativamente ao Desejo de ajudar a sociedade. O mesmo não se verifica para o Desejo de ser professor, onde se encontrou uma diferença significativa entre a área de especialização em Clínica $(\mathrm{M}=.67$, DP $=.914)$ e Não Clínica $(\mathrm{M}=1.14$, DP =1.12); $t(202)=$ $-2.83, p=.02$ ).

Tabela 2.

Comparações de médias (t-Test para amostras independentes) para Psicólogos Clínicos e Não Clínicos, face ao Desejo de ajudar a sociedade e ao Desejo de ser Professor

\begin{tabular}{llcccc}
\hline & & M & DP & $P$ & $T$ \\
\hline${ }^{1}$ Motivação_1 & Clínica & 2.72 & .51 & & \\
& Não & & & .34 & $t_{(202)}=.97$ \\
& Clínica & 2.64 & .57 & & \\
${ }^{2}$ Motivação_18 & Clínica & .67 & .91 & & \\
& Não & & 1.1 & .02 & $t_{(202)=}$ \\
& Clínica & 1.14 & 2 & & -2.83 \\
\hline
\end{tabular}

Nota: ${ }^{1}$ Motivação_1 - Desejo de ajudar a sociedade (item retirado do questionário IBT); ${ }^{2}$ Motivação_18 - Desejo de ser Professor (item retirado do questionário IBT)

Num terceiro grupo de análises, o objetivo passou por explorar o efeito preditivo das Experiências (in Influências -IBT) e da Centralidade do Evento. Do resumo das regressões obtidas tomando esta última como preditor (cf. Tabela 3), é possível averiguar que nem todos os modelos revelam um nível de significância $p$ $<.01$, nomeadamente o primeiro $(p=.35)$, concluindo-se, no entanto, que a Centralidade prediz uma dimensão do IBT (Experiências) e os Sintomas. É também possível analisar que a Centralidade do Evento explica: 6\% dos Sintomas $\left(R^{2}=.06 ; p<.01\right)$ e $12 \%$ da Dimensão Experiências da Escala IBT $\left(R^{2}=.12 ; p<.01\right)$.

\begin{tabular}{cccc}
\hline Modelo & $\mathrm{R}^{2}$ & $\mathrm{R}^{2}$ ajustado & $p$ \\
\hline $1^{\mathrm{a}}$ & .00 & -.01 & .75 \\
$2^{\mathrm{b}}$ & .14 & .13 & $\mathrm{p}<.01^{\mathrm{d}}$ \\
$3^{\mathrm{c}}$ & .04 & .04 & $\mathrm{p}<.01^{\mathrm{d}}$ \\
\hline
\end{tabular}

$\mathrm{Na}$ análise dos coeficientes de regressão, obtidos para cada uma das variáveis dependentes (cf. Tabela 4), foram examinados os valores de Beta $(\beta)$ para a comparação da contribuição da variável independente na variação e explicação das variáveis dependentes (Pallant, 2005). Desta forma, é possível verificar que a Centralidade do Evento não exerce poder preditivo na área de especialização ( $\beta=-.07 ; p=.34)$; no entanto, prediz significativamente os Sintomas $(\beta=.25 ; p<.01)$, e ainda consegue predizer as Experiências $(\beta=.35 ; p<.01)$.

Tabela 3.

Resumo das regressões obtidas para cada variável dependente, com a Centralidade do Evento como preditor

\begin{tabular}{cccc}
\hline Modelo & $\mathrm{R}^{2}$ & $\mathrm{R}^{2}$ ajustado & $p$ \\
\hline $1^{\mathrm{a}}$ & .0 & -.0 & .35 \\
$2^{\mathrm{b}}$ & .06 & .06 & $\mathrm{p}<.01^{\mathrm{d}}$ \\
$3^{\mathrm{c}}$ & .12 & .12 & $\mathrm{p}<.01^{\mathrm{d}}$ \\
\hline
\end{tabular}

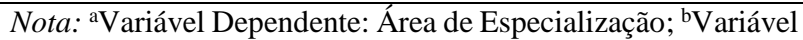
Dependente: Sintomas; 'Variável Dependente: Experiências Total (IBT); ${ }^{\mathrm{d}}$ Preditores: (Constante): Escala da Centralidade do Evento

Tabela 4.

Coeficientes de regressão obtidos para cada variável dependente com a Centralidade do Evento como preditor

\begin{tabular}{ccccc}
\hline Modelo & $B$ & Beta & $\mathrm{T}$ & $\mathrm{P}$ \\
\hline $1^{\mathrm{a}}$ (Constante) & 1.37 & & 13.11 & $\mathrm{p}<.01^{\mathrm{d}}$ \\
ECA_Total & -.0 & -.07 & -.94 & $\mathrm{p}=.34$ \\
$2^{\mathrm{b}}$ (Constante) & 1.76 & & 1.10 & $\mathrm{p}=.27$ \\
ECA_Total & .10 & .25 & 3.66 & $\mathrm{p}<.01^{\mathrm{d}}$ \\
$3^{\mathrm{c}}$ (Constante) & 21.23 & & 7.72 & $\mathrm{p}<.01^{\mathrm{d}}$ \\
ECA_Total & .24 & .35 & 5.28 & $\mathrm{p}<.01^{\mathrm{d}}$ \\
\hline
\end{tabular}

Nota: ${ }^{a}$ Variável Dependente: Área de Especialização; ${ }^{b}$ Variável Dependente: Sintomas; ${ }^{c}$ Variável Dependente: Experiências Total (IBT); ${ }^{d}$ Preditores: (Constante): Escala da Centralidade do Evento

Para a segunda predição (cf. Tabela 5) é possível observar que os modelos, à exceção do primeiro $(p=.75)$, revelam significância estatística. Assim, é possível analisar que as Experiências (IBT) predizem significativamente a Centralidade do Evento e os Sintomas Emocionais Negativos, tendo revelado um nível de significância $p<.01$. É também possível ver que as Experiências explicam $14 \%$ da Centralidade do Evento $\left(R^{2}=.14 ; p<.01\right)$ e $4 \%$ dos Sintomas $\left(R^{2}=.04 ; p\right.$ $<.01)$.

Tabela 5.

Resumo das regressões obtidas para cada variável dependente, com as Experiências como preditor

Nota: ${ }^{a}$ Variável Dependente: Área de Especialização; ${ }^{b}$ Variável Dependente: Centralidade do Evento; ${ }^{\mathrm{c}}$ Variável 
Dependente: Sintomas; ${ }^{d}$ Preditores: (Constante): Experiências Total (IBT)

Na Tabela 6, observam-se os valores de Beta $(\beta)$ para análise da contribuição da variável independente Experiências na variação das dependentes, percebendo-se pelo primeiro modelo que ela não é capaz de predizer por si só a área de especialização $(p>.05)$. Por outro lado, nos modelos seguintes, verifica-se que as Experiências têm uma contribuição considerável na explicação das variáveis dependentes Centralidade do Evento $(\beta=.37 ; p<.01)$ e Sintomas $(\beta=.20 ; p<.01)$.

Tabela 6.

Coeficientes de regressão obtidos para cada variável dependente, com as Experiências como preditor

\begin{tabular}{|c|c|c|c|c|}
\hline Modelo & $B$ & Beta & $\mathrm{T}$ & $\mathrm{p}$ \\
\hline $1^{\mathrm{a}}$ (Constante) & 1.29 & & 24.54 & $\mathrm{p}<.01^{\mathrm{d}}$ \\
\hline Experiências & -.00 & -.02 & -.32 & .75 \\
\hline $2^{\mathrm{b}}($ Constante $)$ & 49.19 & & 24.51 & $\mathrm{p}<.01^{\mathrm{d}}$ \\
\hline Experiências & .85 & .37 & 5.67 & $\mathrm{p}<.01^{\mathrm{d}}$ \\
\hline $3^{c}$ (Constante) & 5.45 & & 6.68 & $\mathrm{p}<.01^{\mathrm{d}}$ \\
\hline Experiências & .179 & .20 & 2.94 & $\mathrm{p}<.01^{\mathrm{d}}$ \\
\hline
\end{tabular}

${ }^{\text {aV}}$ Variável Dependente: Área de Especialização; ${ }^{b}$ Variável Dependente: Centralidade do Evento; ${ }^{c}$ Variável Dependente: Sintomas; ${ }^{d}$ Preditores: (Constante): Experiências Total (IBT)

\section{Discussão e Conclusões}

Tendo em conta os objetivos do presente estudo, foram investigadas as relações entre a Centralidade do Evento, as Experiências e Motivações que influenciam a escolha da carreira em Psicologia, os Sintomas e a Felicidade, considerando a área de especialização, Clínica e Não Clínica. Pretendeu-se igualmente explorar um potencial efeito preditor da Centralidade do Evento traumático e das Experiências. Comentem-se os principais resultados.

Existem algumas semelhanças e diferenças entre os grupos de psicólogos Clínicos e não Clínicos no que se refere às correlações entre a Experiência de ausência ou perda dos pais durante a adolescência/juventude (por exemplo, doença, divórcio, separação, morte), Centralidade do Evento, Sintomas e Felicidade. Para a amostra de Psicólogos Clínicos, verificou-se uma correlação significativa, ainda que baixa, entre a Experiência de Ausência ou Perda dos Pais e a Centralidade do Evento. A subescala Depressão da EADS apresenta uma correlação negativa moderada e significativa com a Felicidade, isto é, quanto mais sintomas Depressivos sentidos, menos níveis de Felicidade serão experienciados. Já as correlações entre a Ansiedade (EADS) e a Felicidade, e entre a Ansiedade e a Centralidade do Evento são significativas, mas baixas, sendo a primeira negativa. Quer isto dizer que, à semelhança do que se verificou aquando da correlação da Depressão e da Felicidade, parece aqui acontecer o mesmo, ou seja, à medida que aumentam os sintomas de Ansiedade, vai diminuir a Felicidade percebida. Também o Stress (EADS) apresenta correlações baixas e estatisticamente significativas com a ESAF e ECA, sendo de notar que a primeira é negativa. Dados provenientes da teoria de Berntsen e Rubin (2006, 2007) sugerem que a memória de um trauma ou evento emocional negativo pode tornar-se central para a vida e identidade do indivíduo, e tal pode estar relacionado com o aumento das reações de stress traumático, depressão e ansiedade. Já na amostra de Psicólogos Não Clínicos, a Experiência e a Felicidade não estabeleceram quaisquer relações, mas verificou-se uma correlação moderada entre a medida total de Sintomas Emocionais Negativos (Escalas de Ansiedade, Depressão e Stress, EADS-21) e a Centralidade do Evento. Mais ainda, a subescala Depressão (EADS) encontra-se moderamente correlacionada com a Centralidade do Evento. Estes dados, a par da teoria de Berntsen e Rubin (2006, 2007), também estão de acordo, em parte, com os estudos de Schore (2001), que preconizam que as experiências adversas podem afetar a maturação e funcionamento dos mecanismos psicobiológicos e influenciar a vulnerabilidade para a psicopatologia.

A Centralidade do Evento traumático não varia segundo a Área de Especialização em Psicologia (Clínica e Não Clínica). Os resultados obtidos revelaram que não existem diferenças estatisticamente significativas ao nível da Centralidade do Evento, segundo a área de especialização. Apesar de alguns estudos apontarem noutro sentido, este dado encontra-se em consonância com a literatura que considera que antecedentes de problemas pessoais não são universais entre pessoas que se tornam profissionais de Psicologia (Murphy \& Halgin, 1995).

Os psicólogos que escolhem a especialização em Clínica, comparativamente com os Não Clínicos, apresentam índices idênticos de Desejo de Ajudar a Sociedade, mas menores quanto ao Desejo de Ser Professor (Motivações). Não se registaram diferenças estatisticamente significativas entre os Psicólogos Clínicos e os Não Clínicos, no que respeita ao Desejo de Ajudar a Sociedade, mas o mesmo não se verifica para o Desejo de ser professor, havendo uma diferença significativa nas pontuações que favorece os Não Clínicos. O resultado que indica diferenças significativas encontra-se em conformidade com Murphy e Halgin (1995), os quais compararam duas amostras, 56 Psicoterapeutas e 53 Psicólogos Sociais, tendo os últimos, não clínicos, reportado mais empenho e entusiasmo no trabalho como professores. Também, os Psicólogos Sociais, quando se referiam à realização profissional e oportunidade, normalmente faziam alusão aos seus interesses no ensino ou na investigação.

A Centralidade do Evento permite predizer Sintomas Emocionais Negativos e Experiências, mas não a Área de Especialização. Apesar de os valores de correlação, atrás mencionados, não terem sido, na sua maioria, muito elevados, fizeram-se análises preditivas com fim exploratório. Das três regressões realizadas, em que a Centralidade do Evento assume o papel de variável independente (preditor), concluiu-se que prediz modestamente uma das dimensões da IBT, as Experiências, e ainda os Sintomas. Com efeito, explica $6 \%$ dos Sintomas e $12 \%$ das Experiências que 
Influenciam a Escolha da Carreira em Psicologia (da Escala IBT).

As Experiências permitem predizer a Centralidade do Evento e os Sintomas, mas não a Área de Especialização. Foi possível constatar que as Experiências que influenciam a escolha da carreira em Psicologia (IBT) predizem significativamente a Centralidade do Evento e os Sintomas, embora explicando apenas 14\% da Centralidade do Evento e 4\% dos Sintomas. Não obstante, Elliott e Guy (1993 citado em Nikčević et. al, 2007) sugeriram que experiências traumáticas na infância podem conduzir, algumas pessoas, a posições de responsabilidade prematura nas suas famílias. No entanto, inesperadamente, foram verificados poucos sintomas de perturbações psicológicas e bons níveis de satisfação interpessoal, sugerindo que um historial familiar problemático não implica, necessariamente, um ajustamento posterior ou uma certa escolha profissional.

Em suma, foi possível observar que antecedentes de problemas pessoais não são universais entre pessoas que se tornam Psicólogos ou que seguem a especialização no ramo de Clínica. Estes resultados vão de encontro a alguns estudos, sugerindo que um histórico familiar problemático não implica, necessariamente, certo tipo de escolha profissional como, por exemplo, a carreira em Psicologia (Murphy \& Halgin, 1995). Também Norcoss e Guy (1989 citados em Murphy \& Halgin, 1995) referem que os terapeutas tendiam a valorizar menos as relações familiares na escolha de carreira e enfatizavam a influência positiva de role models, que os guiavam na iniciação e crescimento profissionais.

Em estudos futuros, sugere-se o alargamento da amostra e um maior equilíbrio quanto à variável sexo, cujo efeito, no que toca às influências na escolha da carreira em Psicologia, será interessante pesquisar. Tão ou mais interessante seria comparar sujeitos de psicologia com outras profissões, fora do campo da Saúde Mental, por exemplo, engenheiros, gestores, etc. Outra questão, para a qual não se procurou neste estudo uma resposta, será, no caso de se provar que acontecimentos adversos na infância levam algumas pessoas a seguir uma carreira em Psicologia, saber se isso terá impacto a nível da prática clínica. Finalmente, outra questão a explorar é saber se os sujeitos que seguem uma carreira em Psicologia, especializando-se em certa área, tiveram ou não algum tipo de barreira no acesso à carreira ou à especialização, como por exemplo, a média de classificação; ou, mesmo, questionar se os sujeitos tinham esta profissão como primeira escolha. O controlo destes aspetos, juntamente com a investigação das questões anteriores, certamente contribuirá para um melhor esclarecimento no âmbito do estudo das influências sobre a escolha de carreira dos psicólogos.

\section{Referências}

Barros, J. (2001). Natureza e Avaliação (Proposta de uma nova Escala). Psic., Ed. e Cultura, 5 (2), 289-318.

Behling, O. \& Law, K. (2000). Translating questionnaires and other research instruments: Problems and solutions (Sage University Papers Series on Quantitative Applications in the Social Sciences, series no. 07-131). Thousand Oaks, CA: Sage.

Berntsen, D. \& Rubin, D. C. (2006). Centrality of Event Scale: A measure of integrating a trauma into one's identity and its relation to post-traumatic stress disorder symptoms. Behav. Research and Therapy, 44, 219-231. http://dx.doi.org/10.1016/j.brat.2005.01.009.

Berntsen, D. \& Rubin, D. C. (2007). When a trauma becomes a key to identity: Enhanced integration of trauma memories predicts posttraumatic stress disorder symptoms. Applied Cognitive Psychology, 21, 417431. http://dx.doi.org/10.1002/acp.1290.

Bluck, S. (2003). Autobiographical Memory: Exploring its Functions in Everyday Life. Hove, England: Psych. Press. http://dx.doi.org/10.1080/741938206.

DiCaccavo, A. (2002). Investigating individuals' motivations to become counselling psychologists: the influence of early caretaking roles within the family. Psychology and Psychoterapy: Theory, Res. and Practice, 75, 463-472. http://dx.doi.org/10.1348/147608302321151943.

Flannery, R. (1999). Psychological Trauma and Posttraumatic Stress Disorder: A Review. Internat J Emergency Mental Health, 2, 135-140. https://www.researchgate.net/publication/12103325_P sychological_trauma_and_posttraumatic_stress_disor der_A_review.

Matos, M., Pinto-Gouveia, J. \& Gomes, P. (2010). A centralidade das experiências de vergonha: Estudo das propriedades psicométricas da versão Portuguesa da Escala da Centralidade do Evento (CES). Psicologia, XXIV, 73-95. http://www.scielo.mec.pt/scielo.php?pid=S0874-2049 2010000100004\&script=sci_abstract.

Murphy, R. A. \& Halgin, R. P. (1995). Influences on the Career Choice of Psychotherapists. Professional Psychology: Research and Practice, 26, 4, 422-426. http://dx.doi.org/10.1037/0735-7028.26.4.422.

Nikčević, A, Kramolisova-Advani, J \& Spada, M (2007). Early Childhood Experiences and Current Emotional Distress: What Do They Tell Us About Aspiring Psychologists? The Journal of Psychology, 141, 1, 25-34. http://dx.doi.org/10.3200/JRLP.141.1.25-34.

Pais-Ribeiro, J., Honrado, A. \& Leal, I. (2004). Contribuição para o estudo da adaptação portuguesa das escalas de Depressão Ansiedade Stress de Lovibond e Lovibond. Psychologica, 36, 235-246. http://www.scielo.mec.pt/pdf/psd/v5n2/v5n2a07.pdf

Pallant, J. (2005). SPSS survival manual: a step by step guide to data analysis using SPSS for Windows (Version 12) (2nd ed.). Austrália: Allen \& Unwin.

Rebelo, L. \& Vale Dias, M. L. (2016). Adaptação portuguesa da escala "Influences on Becoming a Therapist" de Murphy e Halgin - versão de investigação. Documento não publicado. 
Schore, A. N. (2001). The Effects of early relational trauma on right brain development, affect regulation, and infant mental health. Infant Mental Health J, 22, 201-269.

http://dx.doi.org/10.1002/1097-0355(200101/04)22:1 $<201:: A I D-I M H J 8>3.0 . C O ; 2-9$.

\section{Agradecimientos}

Trabalho realizado no âmbito do Instituto de Psicologia Cognitiva, Desenvolvimento Humano e Social (I\&D), FCT (Unidade 192). 